\title{
SELECTED ABSTRACTS FROM THE \\ JOURNAL OF THE EXPERIMENTAL \\ ANALYSIS OF BEHAVIOR, MARCH 1993
}

\section{CONDITIONAL DISCRIMINATION AND EQUIVALENCE RELATIONS: CONTROL BY NEGATIVE STIMULI}

\author{
Cammarie Johnson and MurRay Sidman
}

\author{
NEW ENGLAND CENTER FOR AUTISM
}

\begin{abstract}
Three adult subjects were taught the following two-sample, two-comparison conditional discriminations (each sample is shown with its positive and negative comparison, in that order): A1-B1B2, A2-B2B1; B1-C1C2, B2-C2C1; and C1-D1D2, C2-D2D1. A teaching procedure was designed to encourage control by negative comparisons. Subjects were then tested for emergent performances that would indicate whether the baseline conditional discriminations were reflexive, symmetric, and transitive. The tests documented the emergence of two classes of equivalent stimuli: A1, B2, C1, D2 and A2, $\mathrm{B} 1, \mathrm{C} 2, \mathrm{D} 1$. These were the classes to be expected if the negative comparisons were the controlling comparisons in the baseline conditional discriminations. The negative comparisons, however, were not the comparisons that subjects were recorded as having chosen in the baseline conditional discriminations. Differential test results confirmed predictions arising from a stimulus-control analysis: In reflexivity tests (AA, BB, CC, DD), subjects chose comparisons that differed from the sample; one-node transitivity (AC, BD) and "equivalence" (CA, DB) tests also yielded results that were the opposite of those to be expected from control by positive comparisons; symmetry tests (BA, CB, DC), two-node transitivity (AD) tests, and two-node "equivalence" (DA) tests yielded results that were to be expected from control by either positive or negative comparisons.

Key words: equivalence relations, conditional discrimination, negative stimuli, stimulus control, computer touch screen input, adult humans
\end{abstract}

\section{CHOICE AND DELAY OF REINFORCEMENT: EFFECTS OF TERMINAL-LINK STIMULUS AND RESPONSE CONDITIONS}

\author{
Takashi OMINo and Masato Ito
}

\author{
MEISEI UNIVERSITY AND OSAKA CITY UNIVERSITY
}

\begin{abstract}
In two experiments, pigeons were exposed to concurrent-chains schedules in which a single initiallink variable-interval schedule led to access to terminal links composed of fixed-interval or fixed-delay schedules. In Experiment 1, an 8-s (or 16-s) delay to reinforcement was associated with the standard key, while reinforcer delay values associated with the experimental key were varied from 4 to $32 \mathrm{~s}$. The results of Experiment 1 showed undermatching of response ratios to delay ratios with terminallink fixed-delay schedules, whereas in some pigeons matching or overmatching was evident with the fixed-interval schedules. In Experiment 2, one pair of reinforcer delay values, either 8 versus $16 \mathrm{~s}$ or 16 versus $32 \mathrm{~s}$, was used. In the first condition of Experiment 2, different delays were associated with different keylight stimuli (cued condition). In the second condition, different terminal-link delays were associated with the same stimulus, either a blackout (uncued-blackout condition) or a white key (uncued-white condition). To examine the role of responses emitted during delays, the keys were retracted during a delay (key-absent condition) in the third condition and responses were required by a fixed-interval schedule in the fourth condition. Experiment 2 demonstrated that the choice proportions for the shorter delay were more extreme in the cued condition than in the uncued-blackout condition, and that the response requirement imposed by the fixed-interval schedules did not affect choice of the shorter delay, nor did the key-absent and key-present conditions. These results indicate that the keylight-stimulus conditions affected preference for the shorter of two delays and that the findings obtained in Experiment 1 depended mainly on the keylight-stimulus conditions of the terminal links (i.e., the conditioned reinforcing value of the terminal-link stimuli).

Key words: choice, delay of reinforcement, generalized matching law, conditioned reinforcement, concurrent-chains schedules, fixed-interval schedules, fixed-time schedules, key peck, pigeons
\end{abstract}




\title{
ACQUISITION OF A SPATIALLY DEFINED OPERANT WITH DELAYED REINFORCEMENT
}

\author{
Thomas S. Gritchfield and Kennon A. Lattal
}

\author{
AUBURN UNIVERSITY AND WEST VIRGINIA UNIVERSITY
}

Two experiments investigated the role of an immediate, response-produced auditory stimulus during acquisition, via delayed reinforcement, of a response selected to control for possible unprogrammed, operandum-related sources of response feedback. Experimentally naive rats were exposed to a delayedfood reinforcement condition, specifically a tandem fixed-ratio 1 differential-reinforcement-of-otherbehavior 30-s schedule. The response was defined as breaking a photocell beam located near the ceiling at the rear of the operant conditioning chamber. In Experiment 1, rates of photobeam breaking by each rat increased from near zero, regardless of the presence or absence of a tone that immediately followed the response initiating the delay interval. Though not essential, the tone facilitated response acquisition and resulted in more efficient response patterns at stability. Experiment 2 demonstrated that photobeam-breaking response rates under the delayed reinforcement contingency exceeded those in a preceding baseline condition in which no food was delivered. In addition, upon introduction of the delayed reinforcement procedure, correspondence between response patterns and the requirements of the reinforcement schedule increased over baseline levels in the absence of a food contingency. Together with a previous report of Lattal and Gleeson (1990), the present results suggest that response acquisition with delayed reinforcement is a robust phenomenon that may not depend on a mechanically defined response or an immediate external stimulus change to mediate the temporal gap between response and reinforcer.

Key words: acquisition, reinforcement delay, operant behavior, spatial location, rats

\section{PSYCHOLOGICAL DISTANCE TO REWARD: SEGMENTATION OF APERIODIC SCHEDULES OF REINFORCEMENT \\ Jin-PANG LeUng}

\author{
THE GHINESE UNIVERSITY OF HONG KONG
}

College students responded for monetary rewards in two experiments on choice between differentially segmented aperiodic schedules of reinforcement. On a microcomputer, the concurrent chains were simulated as an air-defense video game in which subjects used two radars for detecting and destroying enemy aircraft. To earn more cash-exchangeable points, subjects had to shoot down as many planes as possible within a given period of time. For both experiments, access to one of two radar systems (terminal link) was controlled by a pair of independent concurrent variable-interval 60-s schedules (initial link) with a 4-s changeover delay always in effect. In Experiment 1, the appearance of an enemy aircraft in the terminal link was determined by a variable-interval (15 s or $60 \mathrm{~s})$ schedule or a two-component chained variable-interval schedule of equal duration. Experiment 2 was similar to Experiment 1 except for the segmented schedule, which had three components. Subjects preferred the unsegmented schedule over its segmented counterpart in the conditions with variable-interval $60 \mathrm{~s}$, and preference tended to be more pronounced with more components in the segmented schedule. These findings are compatible with those from previous studies of periodic and aperiodic schedules with pigeons or humans as subjects.

Key words: choice, psychological distance, interreinforcement interval, aperiodic schedules, concurrent chains, computer game, humans 